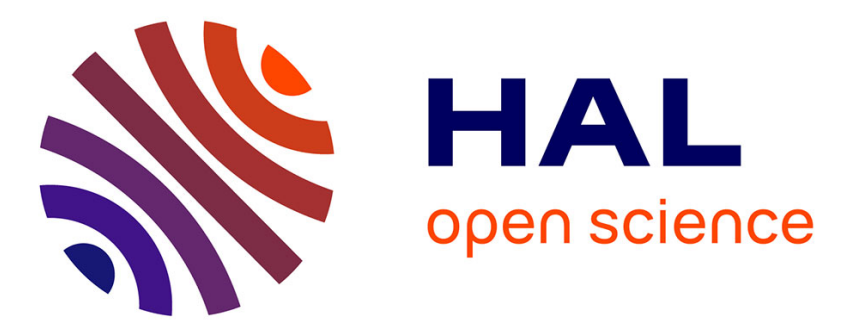

\title{
Fabrication d'un réseau d'interconnexions haute densité pour modules avancés multi-puces par une technique de lift-off
}

J. Palleau, D. Jourdain, J. Oberlin, M. Alaimo, G. Argoud, D. Aubert, M. Beudon, M. Bouzid, D. Laviale, F. Templier, et al.

\section{To cite this version:}

J. Palleau, D. Jourdain, J. Oberlin, M. Alaimo, G. Argoud, et al.. Fabrication d'un réseau d'interconnexions haute densité pour modules avancés multi-puces par une technique de lift-off. Journal de Physique III, 1993, 3 (4), pp.793-804. 10.1051/jp3:1993164 · jpa-00248958

\section{HAL Id: jpa-00248958 https://hal.science/jpa-00248958}

Submitted on 1 Jan 1993

HAL is a multi-disciplinary open access archive for the deposit and dissemination of scientific research documents, whether they are published or not. The documents may come from teaching and research institutions in France or abroad, or from public or private research centers.
L'archive ouverte pluridisciplinaire HAL, est destinée au dépôt et à la diffusion de documents scientifiques de niveau recherche, publiés ou non, émanant des établissements d'enseignement et de recherche français ou étrangers, des laboratoires publics ou privés. 
Classification

Physics Abstracts

81.90

\title{
Fabrication d'un réseau d'interconnexions haute densité pour modules avancés multi-puces par une technique de lift-off
}

\author{
J. Palleau, D. Jourdain, J. C. Oberlin, M. Alaimo, G. Argoud, D. Aubert, M. Beudon, \\ M. J. Bouzid, D. Laviale, F. Templier et J. Torres \\ CNET, B.P. 98, Chemin du Vieux Chêne, 38240 Meylan, France
}

(Reçu le 2 juillet 1992, révisé le 31 décembre 1992, accepté le 20 janvier 1993)

\begin{abstract}
Résumé. - Nous avons développé une filière pour la réalisation de modules multi-chips qui met en œuvre le cuivre comme conducteur, un polymère entièrement cyclisé comme diélectrique et utilise les bonnes propriétés conductrices d'un substrat de silicium fortement dopé permettant son utilisation comme plan de masse. Après une présentation des objectifs assignés à une telle filière, visant la réalisation de modules multi-chips pour des systèmes dédiés aux télécommunications, nous développerons la technique du lift-off utilisée pour la réalisation des différents niveaux d'interconnexions, ainsi que les techniques élémentaires de gravure et de métallisation. Dans un dernier point nous analyserons les principales caractéristiques physiques, mécaniques et électriques de tels réseaux.
\end{abstract}

\begin{abstract}
We have developed technological processes to assemble interconnect networks for multichip modules (MCM). Copper is used as conductor material. A new polymeric material, fully cyclised in solution, is used as dielectric. The multi-layered structure is deposited on highly doped silicon substrate used as ground plane. We present several physical, mechanical and electrical characterizations performed all along the network assembling.
\end{abstract}

\section{Une filière MCM pour télécommunication ?}

Le domaine des télécommunications est particulièrement sensible aux problèmes de limitation en fréquence, notamment depuis l'apparition des Réseaux Numériques à Intégration de Services (RNIS). En effet, pour ce type de réseau, les informations numérisées sont transmises à des fréquences de 16 kbits à 2 Mbits [1]. Or, la prochaine étape dans l'évolution des réseaux de télécommunications avec la création du RNIS large bande requiert des densités de transmission d'information de l'ordre du Gbits pour être compatible avec les vidéo-communications dont la TVHD. Pour pouvoir développer les systèmes de télécommunications de demain, mais aussi tous les systèmes rapides, on doit impérativement s'affranchir des limitations, tant en densité d'intégration qu'en fréquence, imposées par les interconnexions entre puces. De tels dispositifs, déjà développés depuis quelques années, nommés structures multi-puces, ont permis un gain important sur les performances des systèmes. Mais seul le 
passage aux structures couches minces permettra d'atteindre les gains requis, tant en vitesse qu'en densité d'intégration. Il apparaît sur le tableau I que pour une complexité de module donnée (100 circuits intégrés), le nombre de couches augmente avec le nombre d'entrées/sorties par circuit intégré [2].

Tableau I. - Comparaison de réseaux réalisés en couches minces et en couches épaisses. [Comparison of thin film and thick film networks.]

\begin{tabular}{|c|c|c|c|c|}
\hline E-S puce & Puce/module & Surf./puce $\mathrm{cm}^{2}$ & $\begin{array}{c}\text { Nb. couches } \\
\text { film épais }\end{array}$ & $\begin{array}{c}\text { Nb. couches } \\
\text { film mince }\end{array}$ \\
\hline 158 & 100 & 1 & 12 & 2 \\
\hline 239 & 100 & 1 & 18 & 2 \\
\hline 849 & 100 & 1 & 41 & 4 \\
\hline 1262 & 100 & 1 & 62 & 6 \\
\hline 1262 & 100 & 1 & 95 & 4 \\
\hline 1913 & 100 & 1 & 95 & 12 \\
\hline 1913 & 100 & 1 & 144 & 6 \\
\hline
\end{tabular}

Couches épaisses $=10 \mu \mathrm{m}$ de large, $350 \mu \mathrm{m}$ de pas.

Couches minces $=12 \mu \mathrm{m}$ de large, $25 \mu \mathrm{m}$ de pas.

Couches très minces $=6 \mu \mathrm{m}$ de large, $12 \mu \mathrm{m}$ de pas.

Pour des règles de dessin classiques en technologie couches épaisses (sérigraphie), le nombre de couches empilées atteint rapidement les limites du savoir-faire actuel, soit environ une cinquantaine de couches. A cela s'ajoute une complexité de routage augmentant de manière rédhibitoire le temps et les difficultés de conception. La diminution des dimensions permet d'augmenter la densité d'interconnexion par niveau et de diminuer le nombre des couches empilées. Ainsi en diminuant par un facteur 10 la largeur des lignes et leurs espacements on divise par ce même facteur 10 le nombre de couches empilées. Par exemple pour le module de 100 puces du tableau 1 , quand le nombre d'entrées/sorties par puce est de 1900 , le réseau d'interconnexion nécessite 144 couches dans la technologie couches épaisses, 12 dans la technologie couches minces et 6 dans la technologie couches très minces.

\section{Les règles de dessin.}

La filière développée par le CNET est une filière à haute densité du type Multi-chips Module, (MCM), utilisant une technologie couches très minces. Les règles de dessin utilisées dans cette filière, $10 \mu \mathrm{m}$ pour les largeurs de lignes espacées de $10 \mu \mathrm{m}$, des dimensions de piliers de 
$10 \mu \mathrm{m}$ de diamètre, conduisent à une densité de lignes de $500 \mathrm{~cm} / \mathrm{cm}^{2}$. L'isolement entre masse et alimentation est réalisé par un oxyde thermique $\left(\mathrm{SiO}_{2}\right)$ de $200 \mathrm{~nm}$ ce qui permet d'obtenir une très forte capacité de découplage entre ces deux plans. L'isolement entre les autres niveaux actifs est réalisé par un diélectrique organique de $2,5 \mu$ d'épaisseur.

\section{Les matériaux.}

Pour obtenir des MCM présentant des caractéristiques adaptées aux dimensions et aux utilisations visées, nous avons choisi le cuivre comme matériau conducteur et pour diélectrique un diélectrique polymère. Le support de réseau est un substrat silicium.

3.1 LE CHOIX DU SUBSTRAT. - Celui-ci est guidé par les considérations suivantes : bonne tenue mécanique, bonne conductivité thermique, compatibilité entre les coefficients de dilatation du substrat et des circuits intégrés reportés sur le réseau. Le silicium apparaît être un bon compromis. Sa tenue mécanique est acceptable, sa conductivité thermique est meilleure que celle de l'alumine et il a le même coefficient de dilatation que les circuits intégrés VLSI ou ULSI réalisés sur silicium. Son coefficient de dilatation est aussi très proche de celui de GaAs (Tab. II).

Tableau II. - Comparaison des propriétés des matériaux utilisés comme support. [Comparison of materials properties for different substrates.]

\begin{tabular}{|l|c|c|c|}
\hline & $\begin{array}{c}\text { Coef. expansion } \\
\text { thermique } \\
\left(\mathrm{ppm} /{ }^{\circ} \mathrm{C}\right)\end{array}$ & $\begin{array}{c}\text { Conduct. } \\
\text { thermique } \\
\left(\mathrm{W} / \mathrm{M}^{\circ} \mathrm{C}\right)\end{array}$ & $\begin{array}{c}\text { Constante } \\
\text { diélectrique } \\
(1 \mathrm{MHz})\end{array}$ \\
\hline Silicium & 2,6 & 147 & 11,9 \\
\hline Alumine & 6,5 & 17 & 10,2 \\
\hline Nitrure d'Al & 4,5 & 150 & 10 \\
\hline
\end{tabular}

3.2 LE CHOIX DU MATÉRIAU DIÉLECTRIQUE. - Les critères suivants nous ont conduit à choisir le polyphénylquinoxaline (PPQ).

- Propriétés diélectriques : avec une valeur d' $\varepsilon_{\mathrm{r}}$ de 2,7 et un $\operatorname{tg} \delta$ de $5 \times 10^{-4}$ valeurs déterminées à $1 \mathrm{kHz}$ ce matériau se présente comme un des meilleurs matériaux disponibles aujourd'hui sur le marché.

- Propriétés thermo-mécaniques. Ce matériau thermostable, entièrement cyclisé, présente d'indiscutables facilités de stockage et d'utilisation par rapport aux polyimides classiques.

Enfin son $T_{\mathrm{g}}$ élevé, $360^{\circ}$, présente entre autre avantage, celui de pouvoir réaliser des traitements thermiques prolongés sans que le matériau ne présente un taux de réticulation important et donc une dégradation des propriétés visco-élastiques. 
Le PPQ, fourni par le CEMOTA, est disponible sous forme de solution à $13,5 \%$ dans un mélange de métacrésol/xylène $35 / 65$ présentant une viscosité dynamique de $1600 \mathrm{mPa}$.S. La réalisation de couches minces par spin-coating dans une gamme $2 \mu \mathrm{m}$ à $5 \mu \mathrm{m}$ peut être réalisée par un simple ajustement de la vitesse de rotation. Pour des épaisseurs inférieures à $2 \mu \mathrm{m}$ il est nécessaire d'ajuster la viscosité par dilution dans le mélange de solvants. Les traitements thermiques des films, fixés par le fabricant, consistent en un recuit étagé permettant d'obtenir des couches homogènes en contrainte et permettant l'élimination des solvants résiduels. La température maximum est limitée à $T_{\mathrm{g}}$ pour éviter une dégradation de la limite de déformation plastique qui, dans ces limites thermiques, est de l'ordre de $8 \%$ à $12 \%$.

3.3 LE CHOIX DU MATÉRIAU CONDUCTEUR. - Le critère déterminant pour le choix du matériau conducteur est bien sûr la résistivité. C'est donc ce critère qui a déterminé notre choix. Le cuivre déposé par pulvérisation cathodique permet d'obtenir de façon reproductible un matériau présentant une résistivité proche de celle du cuivre massif soit $1,7 \mu \Omega \mathrm{cm}^{-1}$ Pour la gravure du cuivre, seuls les procédés de gravure liquide sont maîtrisés mais ne peuvent conduire à des définitions de motifs compatibles avec les dimensions visées. En raison de cette limitation nous avons retenu la technique du lift-off, technique nous permettant de nous affranchir de toute contrainte dimensionnelle.

\section{Les technologies élémentaires.}

Les règles de dessin visées, la complexité des circuits à réaliser, passent inévitablement, pour la réalisation, par l'utilisation des technologies de la micro-électronique.

4.1 LE LIFT-OFF. - La technique du lift-off, qui a été très largement utilisée par le constructeur IBM [3] pour la réalisation du dernier niveau d'interconnexions sur le circuit intégré, présente ici le double avantage de s'affranchir du problème de la gravure du cuivre et de réaliser une planarisation niveau par niveau avec des variations de planéité ne dépassant pas quelques centaines de nanomètres.

Principe : la technologie développée par le CNET s'appuie sur le principe du lift-off assisté mécaniquement (brevet CNET) [4].

Ce lift-off repose sur une structure triple couches :

- la couche de résine photo-sensible ;

- une couche de SOG utilisée là comme contre masque permettant de transférer par gravure plasma l'image du motif dans la couche de diélectrique ;

- une couche de lift-off proprement dit, réalisée en un matériau facilement soluble dans un solvant organique.

Le mécanisme se résume en cinq étapes (Fig. 1) :

1) le transfert de l'image du masque dans la résine photosensible par insolation;

2) le transfert de l'image de la résine photosensible dans le contre masque par gravure RIE ;

3) l'ouverture du diélectrique dans les zones destinées à recevoir le conducteur ;

4) la métallisation;

5) le retrait du métal des zones non électriquement actives.

Le choix des matériaux est déterminant dans la réalisation d'une telle structure puisque c'est par relaxation des contraintes que la plus grande partie du travail de lift-off sera réalisée.

La couche de lift-off. - La couche de lift-off est une couche de matériau polymère, soluble dans un solvant organique spécifique, n'ayant aucune action sur la couche diélectrique PPQ. 


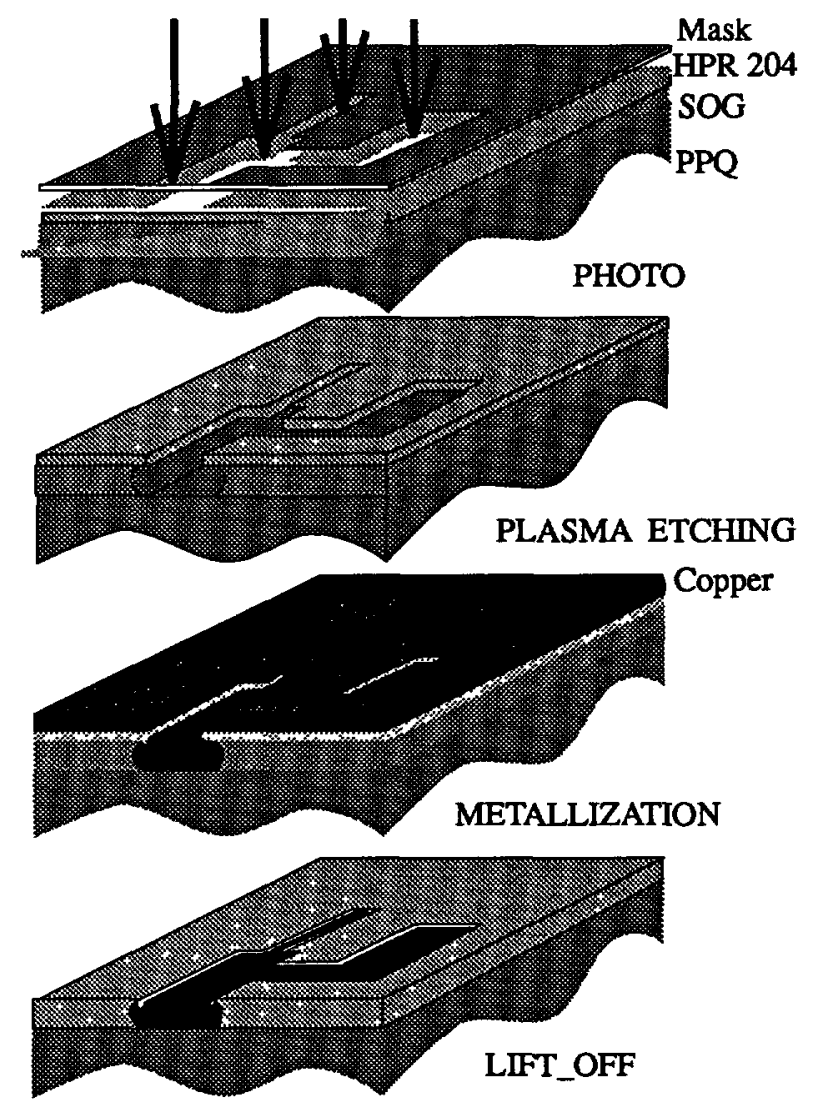

Fig. 1. - Les étapes du lift-off.

[The steps of the lift-off process.]

Ce matériau de lift est en cours de développement chez le fabricant CEMOTA. Sa température d'utilisation se situe dans la gamme $200^{\circ} \mathrm{C}, 250^{\circ} \mathrm{C}$.

Le contre masque. - Le rôle du contre masque est uniquement d'apporter une grande sélectivité de gravure entre les zones de diélectrique recouvertes de résine photosensible et celles révélées. La résine photosensible qui présente une vitesse de gravure équivalente à celle du PPQ ne permet pas d'obtenir une telle sélectivité. La gravure de ce contre masque est réalisée en plasma fluoré, conditions pour lesquelles la sélectivité SOG/HPR est très grande. Dans ce cas particulier d'utilisation les températures de recuit des SOG peuvent être fortement diminuées et se situer dans une gamme $200^{\circ} \mathrm{C}, 250^{\circ} \mathrm{C}$ tout en respectant d'excellentes conditions de sélectivité en gravure.

L'application de la technologie de lift-off à la réalisation de motifs présentant des épaisseurs de quelques microns demande une bonne maîtrise de la métallisation qui doit être suffisamment directive pour qu'une discontinuité soit respectée entre la partie supérieure de la métallisation et les motifs. Cette discontinuité facilite de plus la pénétration du solvant vers la couche organique de lift-off. 
4.2 LE DÉPÓT PAR PULVÉRISATION MAGNÉTRON. - La métallisation réalisée par pulvérisation magnétron, bien que le dépôt soit peu directionnel, présente l'avantage de vitesses de dépôt importantes, supérieures d'un ordre de grandeur aux vitesses de dépôt par évaporation. La directionnalité peut par ailleurs être sensiblement améliorée en optimisant les paramètres du procédé : pression du plasma et distance cible/substrat.

La dégradation possible des interfaces, à partir de faibles traces d'oxygène résiduel dans les atmosphères de recuit conduit à la formation d'oxyde de cuivre. La dégradation catalytique du polymère observée en présence d'oxyde de cuivre [5], nous a conduit à encapsuler les motifs métalliques d'une couche de chrome de $50 \mathrm{~nm}$ à $100 \mathrm{~nm}$ [5]. Un bâti de pulvérisation multicibles et multiplaques permet de réaliser en une seule étape l'ensemble des dépôts avec des vitesses de dépôt pouvant atteindre le micron/minute pour le cuivre.

Tableau IIIa. - Vitesses de gravure des différents matériaux.

[Etching rates for different materials.]

\begin{tabular}{|l|c|c|c|c|}
\hline $\begin{array}{c}\text { Vitesses } \\
\mathrm{Nm} / \mathrm{mn}\end{array}$ & $\mathrm{V} / \mathrm{O}_{2}$ & $\begin{array}{c}\mathrm{V} / \mathrm{O}_{2}+ \\
\mathrm{C}_{2} \mathrm{~F}_{6}\end{array}$ & $\begin{array}{c}\mathrm{V} / \mathrm{CHF}_{3}+\mathrm{C}_{2} \mathrm{~F}_{6} \\
+\mathrm{He}\end{array}$ & $\mathrm{V} \downarrow / \mathrm{V} \rightarrow$ \\
\hline $\mathrm{PPQ}$ & 150 & 183 & 0,7 & 8 \\
\hline $\mathrm{SOG}$ & 0,5 & 29 & 40 & $>100$ \\
\hline $\mathrm{SiO}_{2}$ Ther. & & 12 & 16 & \\
\hline $\mathrm{SiO}_{2}$ pulvé & & & 160 & \\
\hline $\mathrm{HPR}$ & 160 & 175 & & \\
\hline
\end{tabular}

Tableau IIIb. - Sélectivité selon les conditions de gravure.

[Etching selectivity obtained by RIE for different materials.]

\begin{tabular}{|l|c|c|c|}
\hline \multicolumn{1}{|c|}{$\begin{array}{c}\text { Sélectivite } \\
\text { Sélectivité }\end{array}$} & $\mathrm{O}_{2}$ & $\mathrm{O}_{2}+\mathrm{C}_{2} \mathrm{~F}_{6}$ & $\begin{array}{c}\mathrm{CHF}_{3}+ \\
\mathrm{C}_{2} \mathrm{~F}_{6}+\mathrm{HE}\end{array}$ \\
\hline $\mathrm{PPQ} / \mathrm{SOG}$ & 80 & 1,4 & 1 \\
\hline $\mathrm{HPR} / \mathrm{SOG}$ & & 6 & 2,5 \\
\hline $\mathrm{PPQ} / \mathrm{SiO}_{2}$ pulvé. & & & 23 \\
\hline $\mathrm{PPQ} / \mathrm{SiO}_{2}$ ther. & & 15 & 36 \\
\hline $\mathrm{SOG} / \mathrm{SiO}_{2}$ pulvé. & & & 2,5 \\
\hline $\mathrm{SOG} / \mathrm{SiO}_{2}$ ther. & & & 1,6 \\
\hline
\end{tabular}


4.3 LA GRAVURE RIE. - Les gravures des différents matériaux sont réalisées en mode RIE. Les gaz de gravure utilisés sont de deux types : l'oxygène pour le diélectrique et un mélange $\mathrm{C}_{2} \mathrm{~F}_{6} / \mathrm{He}$ pour le SOG, l'oxyde thermique et les couches d'arrêt. Pour assurer une meilleure discontinuité entre les parties métalliques déposées sur le SOG et les motifs, un profil particulier, en surplomb, est réalisé par gravure isotrope du diélectrique (Fig. 2). Les vitesses de gravure, les sélectivités et les rapports d'anisotropie sont donnés dans les tableaux IIIa et IIIb.

Bien que ces différents procédés de gravure soient parfaitement maîtrisés, ils ne permettent pas, compte tenu des dispersions sur l'épaisseur des couches déposées et des inhomogénéités des vitesses de gravure liées à la dispersion des dimensions des motifs, d'obtenir des profondeurs de motifs rigoureusement identiques. Cette difficulté peut être levée par l'utilisation d'une couche d'arrêt. Celle-ci bloquant sur le même plan la gravure de tous les motifs, ils seront positionnés à la même altitude quel que soit le temps de surgravure nécessaire pour obtenir le profil le plus adapté au remplissage. La surgravure des fonds de trous dans les zones de contact permet aussi de minimiser les résistances de contact. Cette couche d'arrêt qui doit présenter une bonne sélectivité par rapport à la vitesse de gravure du PPQ est une couche mince de $\mathrm{SiO}_{2}$ de $100 \mathrm{~nm}$ à $150 \mathrm{~nm}$ déposée par pulvérisation magnétron. L'encapsulation du diélectrique par cette couche de $\mathrm{SiO}_{2}$ présente en outre l'avantage de limiter fortement les reprises de solvant et d'humidité par le diélectrique.

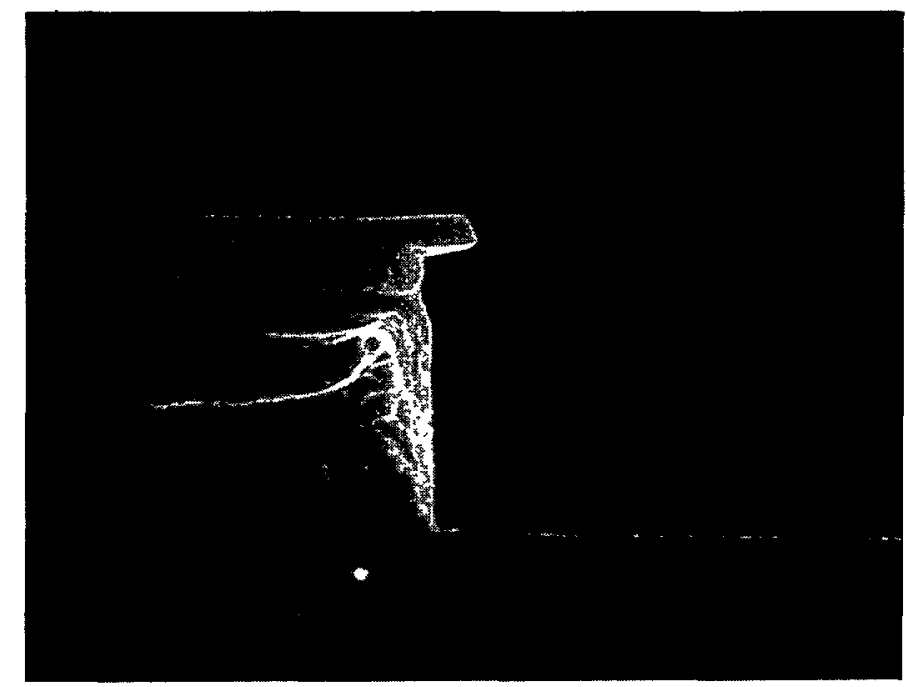

Fig. 2. - Profil de SOG sur polymère obtenu par gravure RIE.

[SOG on polymer profil obtained by RIE.]

\section{Les filières du CNET.}

Sur la base de ces technologies élémentaires deux types de filières sont permises.

La filière Si non dopé : réalisée sur un substrat de Si servant uniquement de support isolé des plans supérieurs par un niveau de diélectrique. Cette technologie n'utilise pas les potentialités du substrat silicium. Une telle filière a été réalisée sur substrat d'alumine [6]. 
La filière Si dopé : cette filière utilise les propriétés conductrices du silicium fortement dopé pour la réalisation du plan d'alimentation. Elle permet de plus d'utiliser l'oxyde de silicium pour réaliser les capacités de découplage entre masse et alimentation (Fig. 3). C'est cette filière que nous avons développée au CNET sur substrats de $100 \mathrm{~mm}$.

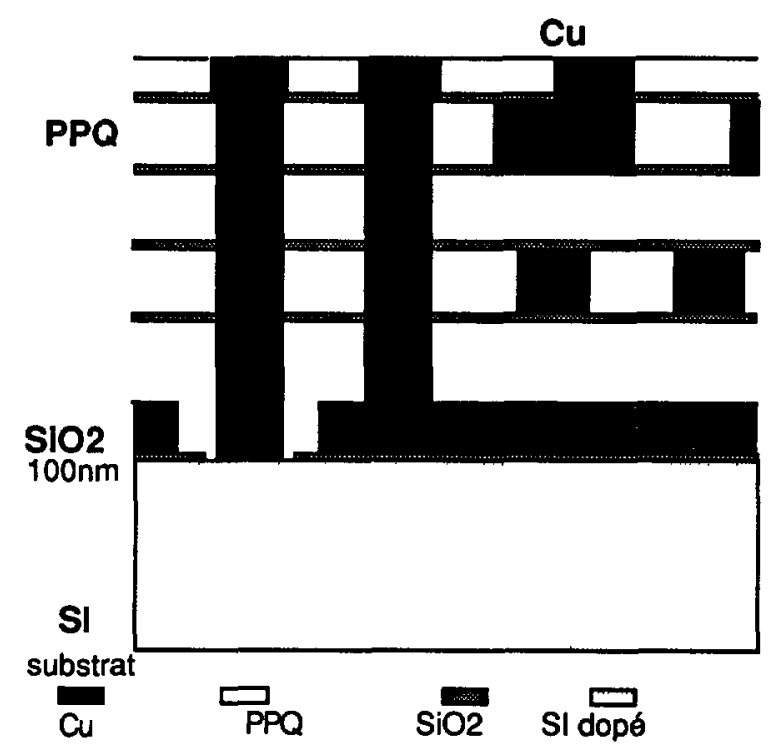

Fig. 3. - Schéma de principe d'un réseau MCM sur silicium dopé.

[Schematic representation of MCM substrat on doped silicon.]

5.1 OPTIMISATION, CARACTÉRISATION DES PARAMÈTRES DE PROCÉDÉS. - L'optimisation des paramètres de mise en œuvre du matériau diélectrique (temps et température de recuit) vise à obtenir, pour une courbure minimum des substrats, les meilleures conditions d'adhérence polymère/silicium et polymère/métal ; les qualités intrinsèques des matériaux (diélectrique et conducteur) et des interfaces étant par ailleurs conservées.

Pour des conditions de mise en œuvre fixees à $300^{\circ} \mathrm{C}$ pendant $2 \mathrm{~h}$ avec recuit intermédiaire à $250{ }^{\circ} \mathrm{C}$ les valeurs des courbures observées (mesure de la flèche sur un diamètre, soit $100 \mathrm{~mm}$ ), pour un premier niveau intégrant l'ensemble du procédé diélectrique et conducteur, sont comprises entre $5 \mu \mathrm{m}$ et $10 \mu \mathrm{m}$.

Les valeurs d'adhérence mesurées par «peel test " sont de $180 \mathrm{~g} / \mathrm{cm}$ à $220 \mathrm{~g} / \mathrm{cm}$.

Les planéités mesurées (hauteur de marche entre le diélectrique et les parties métalliques) sont inférieures à $200 \mathrm{~nm}$.

Aucun gonflement du polymère, lié à une absorption de solvant n'a été observé, résultat qui est confirmé par l'absence, caractérisée par spectrographie infrarouge, de traces de solvant résiduel dans la couche de diélectrique.

La stabilité des interfaces métal/diélectrique, testée après cyclage thermique, ne laisse apparaître aucune dégradation chimique ou mécanique confirmant ainsi les résultats [5] sur le rôle du chrome comme barrière de diffusion de l'oxygène. 
5.2 Caractérisation de l'assemblage. - Comme nous pouvons le voir sur la figure 3 la réalisation d'un réseau nécessite sept niveaux, chacun reprenant toutes les opérations élémentaires précédemment décrites, photo, gravure, métallisation, lift-off.

Les tests reconduits au terme de l'ensemble des étapes, conduisant à la réalisation du module, confirment les résultats obtenus sur les étapes élémentaires.

5.2.1 Caractéristiques mécaniques du réseau. - La courbure des substrats : celle-ci n'est pas cumulative avec le nombre de couches empilées. Les valeurs observées, en fin d'assemblage, sont comprises entre $30 \mu \mathrm{m}$ et $40 \mu \mathrm{m}$.

Les valeurs d'adhérence n'ont pu être mesurées pour les empilements diélectrique/couche d'arrêt/diélectrique. En effet les charges exercées sans que l'on puisse observer de délamination sont celles correspondant à la charge à la rupture du film de polymère.

La planarisation si elle a tendance à se dégrader au cours de l'empilement des différents niveaux reste dans la limite de $250 \mathrm{~nm}$. Ni les propriétés intrinsèques des matériaux (résistivités, constante diélectrique) ni les propriétés d'interface ne se dégradent au cours de réalisation des différents niveaux.

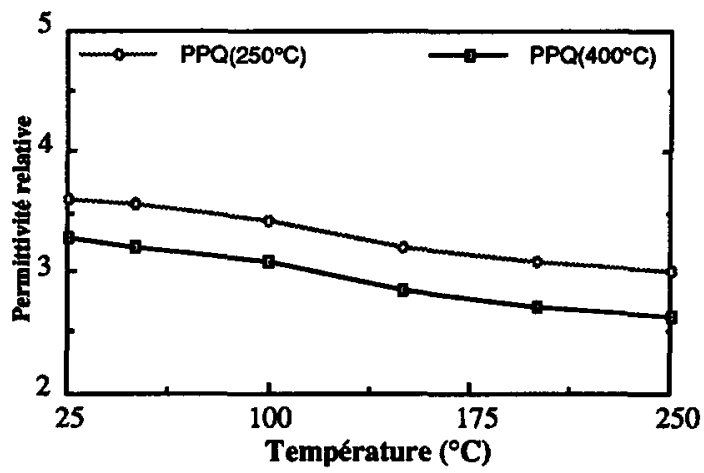

Fig. 4. - Constante diélectrique du PPQ après recuit à différentes températures.

[PPQ dielectric constant for several annealing temperatures.]

5.2.2 Caractéristiques électriques. - La caractérisation électrique du matériau diélectrique. Les mesures de constante diélectrique, de tangente de perte et de tension de claquage, ont été réalisées dans un banc de mesure spécifique. Ce banc permet d'effectuer des mesures sous vide afin de quantifier l'influence, sur les paramètres diélectriques, des différents solvants et de l'eau absorbée en cours du procédé. On peut aussi travailler sous atmosphère contrôlée et, grâce à une sole chauffante, assurer la totale désorption des solvants.

Sur des motifs de tests paramétriques, nous avons suivi la mise au point de la filière, niveau par niveau. Ces motifs de tests comportent :

- des condensateurs, réalisés avec $\mathrm{SiO}_{2}$ thermique comme diélectrique au premier niveau, avec le PPQ aux niveaux supérieurs :

- des résistances de longueur de lignes de $100 \mathrm{~mm}$ et de largeur de $5 \mu \mathrm{m}, 10 \mu \mathrm{m}$ et $20 \mu \mathrm{m}$. Elles permettent de mesurer la résistivité du matériau cuivre en couches minces et de vérifier la qualité de remplissage des lignes en fonction de leur largeur :

- un motif de résistance de contact faisant intervenir jusqu à 6000 contacts. 
Sur ces motifs les résultats suivants ont été obtenus :

Mesures capacitives :

Capacités de découplage en $\mathrm{SiO}_{2}$ thermique :

$$
\begin{aligned}
& \text { épaisseur }=100 \mathrm{~nm} \\
& \text { rigidité diélectrique }=5 \mathrm{MV} / \mathrm{cm} \\
& \text { capacité }=32 \mathrm{nF} / \mathrm{cm}^{2} \\
& \varepsilon_{\mathrm{r}}=4 .
\end{aligned}
$$

Capacités entre niveaux conducteurs :

$$
\begin{aligned}
& \text { épaisseur }=2,5 \mu \mathrm{m} \\
& \text { rigidité diélectrique moyenne }=1 \mathrm{MV} / \mathrm{cm} \\
& \text { capacité }=1,2 \mathrm{nF} / \mathrm{cm}^{2} \\
& \varepsilon_{\mathrm{r}}=3,3 \\
& \operatorname{tg} \delta=1 \times 10^{-3}
\end{aligned}
$$

Résistance de lignes de longueur $110 \mathrm{~mm}$ :

lignes de $5 \mu \mathrm{m}$,

$$
R=16,84 \Omega
$$

lignes de $10 \mu \mathrm{m}$,

$$
R=8,62 \Omega
$$

lignes de $20 \mu \mathrm{m}$,

$$
R=4,21 \Omega \text {. }
$$

Soit :

résistivité moyenne $\mathrm{Cu}=1,92 \mu \Omega . \mathrm{cm}$.

La valeur de résistivité du cuivre trouvée sur ces couches minces est très voisine de la valeur connue pour le matériau massif, soit $1,7 \mu \Omega$.cm.

Résistances de contact.

Bonne ouverture des contacts par gravure.

Chaînes de 6000 contacts passante.

$R_{\mathrm{c}}=10^{-2} \Omega$ par contact de $100 \mu \mathrm{m}^{2}$

La figure 5a représente la photo du motif chaîne de contacts avec une série de sorties intermédiaires, permettant de mesurer un nombre croissant de contacts sur la chaîne. La vue $5 b$ montre le détail de la réalisation : les tronçons de lignes sur deux niveaux étant reliés par des piliers.

Le comportement en propagation de lignes parallèles réalisées dans nos règles de dessin a été étudié. Les lignes ont été modélisées et simulées en utilisant le simulateur PATRICE, développé au LEMO. La simulation a été validée expérimentalement en utilisant des véhicules de tests réalisés au CNET. Les motifs de test sont constitués d'un ensemble de lignes de $50 \mathrm{~mm}$ de long. Ils comportent des lignes isolées, deux ensembles de 2 lignes couplées espacées soit de $10 \mu \mathrm{m}$ soit de $30 \mu \mathrm{m}$, un ensemble de 3 lignes couplées espacées de $10 \mu \mathrm{m}$. La mesure a été réalisée en utilisant un banc d'analyse temporelle, les signaux étant propagés sur des câbles coaxiaux. Il a été done nécessaire de réaliser un montage de test assurant la transition entre le câble coaxial et la ligne micro-ruban. Le rôle de ce système est de réaliser le passage entre des éléments ayant des dimensions géométriques très différentes, tout en gardant une impédance caractéristique constante. Il a été utilisé pour cela un guide planaire à fentes couplées, bien adapté à ce genre de transition. La figure 6 montre une vue du dispositif. La transition ligne coaxiale - guide planaire $50 \Omega$ est assurée par des connecteurs coaxiaux SMA, soudés à une extrémité du réseau des lignes coplanaires. L'autre extrémité du réseau des lignes coplanaires est connectée aux motifs à tester par des fils d'or soudés par ultrason. Le dispositif est alimenté par un générateur de commande dont le temps de montée est de 24 ps et d'amplitude $500 \mathrm{mV}$. 


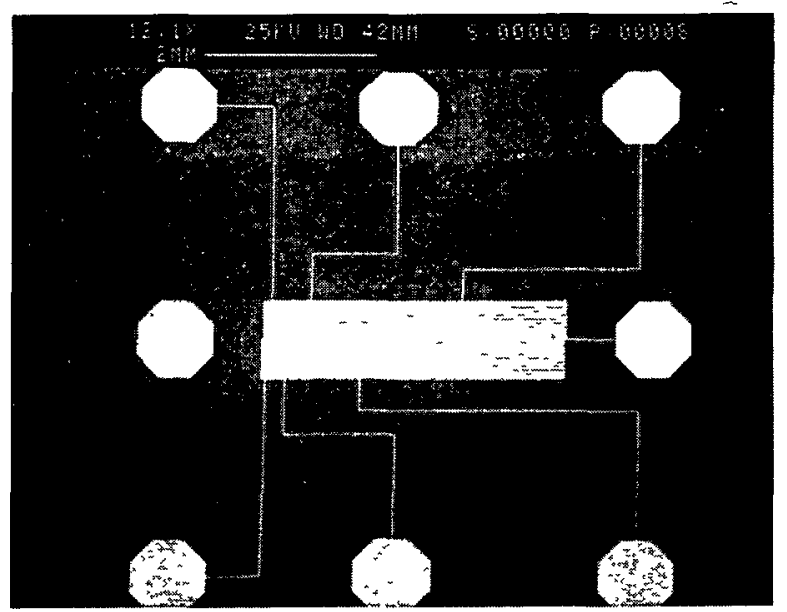

a)

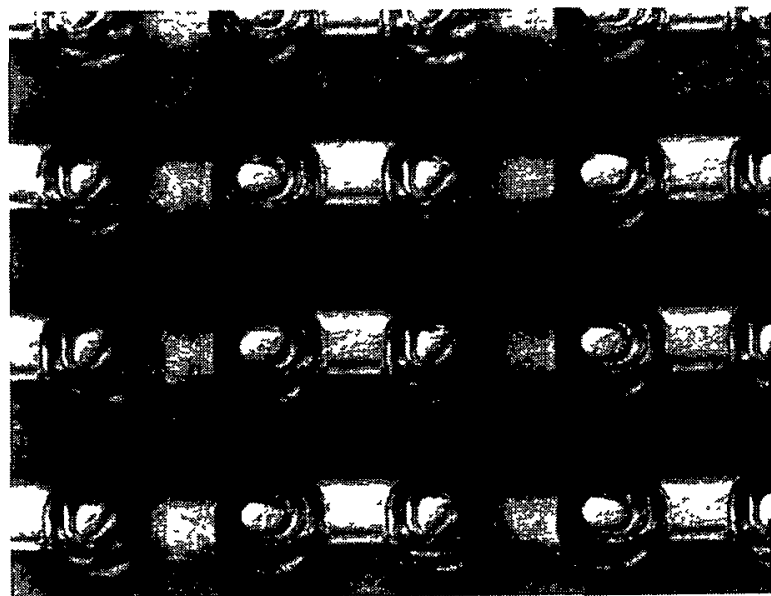

b)

Fig. 5. - a) Chaîne de contacts, vue d'ensemble. b) Chaîne de contacts, $10 \mu \mathrm{m}$ au pas de $20 \mu \mathrm{m}$. [a) Contact chain, general view. b) Contact chain, $10 \mu \mathrm{m}$ at pitch of $20 \mu \mathrm{m}$.]

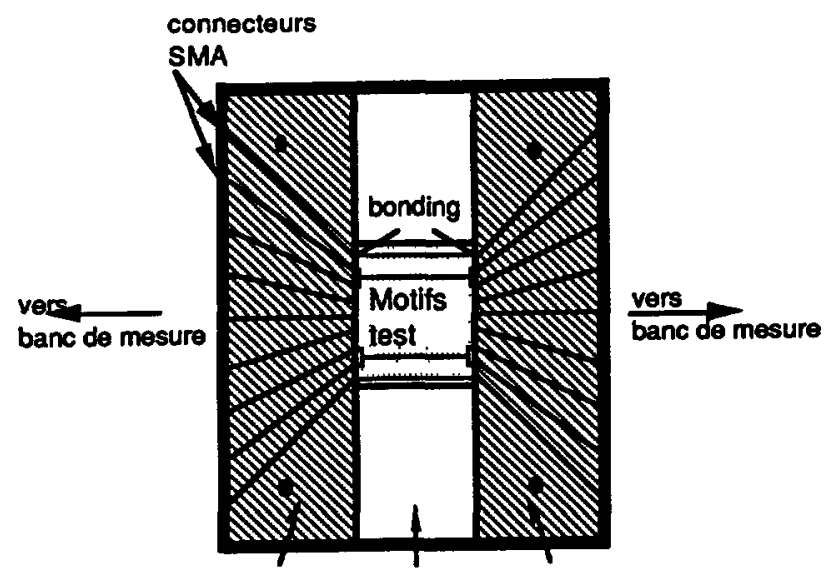

Fig. 6. - Schéma de principe du module de test.

[Schematic representation of the test module.] 
Il a été trouvé :

- une atténuation de $0,75 \mathrm{~dB} / \mathrm{cm}$ à $500 \mathrm{MHz}$;

- un couplage entre lignes séparées de $10 \mu \mathrm{m}$ de l'ordre de $5 \%$;

- un couplage entre lignes espacées de $30 \mu \mathrm{m}$ de l'ordre de $1,5 \%$.

Les règles de dessin choisies sont donc satisfaisantes. Elles sont validées jusqu'à une fréquence d'horloge de $500 \mathrm{MHz}$.

\section{Conclusion.}

Nous avons décrit dans ce travail les différentes étapes suivies pour la mise au point d'une filière d'assemblage de MCM réalisés en couches minces, polymère/métal, sur silicium. La démarche passe par une optimisation des étapes élémentaires avant de prendre en compte les problèmes spécifiques liés à l'assemblage.

Ainsi nous avons pu maîtriser les différentes interfaces du point de vue de leur tenue aux contraintes thermiques, mécaniques et chimiques tout en respectant l'intégrité des propriétés physiques des matériaux mis en œuvre.

Puis à partir d'une analyse rigoureuse des phénomènes observés sur les étapes élémentaires nous avons procédé à l'assemblage complet de la filière.

La réalisation de véhicules de test a permis de valider la filière d'assemblage et les règles de dessin choisies.

La fonctionnalité des véhicules de test, leurs performances obtenues placent cette filière parmi les plus performantes actuellement opérationnelles.

\section{Bibliographie}

[1] OHSAKI T., IEEE, Trans. Components, Hybrids Manuf. Tech. 14 (1991) 254-61.

[2] Rao, Tummala R., Eugene Rymaszewski J., Microelectronics Packaging Handbook (Reinhold Edition, New York, 1989).

[3] International Business Machines Corporation, Structures métalliques à niveaux multiples et procédé de fabrication de celle-ci, Brevet $\mathrm{N}^{\circ} 84115496.6$ (14/12/84).

[4] Schiltz A., Palleau J., Torres J., Brevet N 9002690 (23 février 1990).

[5] Even R., Palleau J., Oberlin J. C., Pantel R., Lavial D., Templier F., Torres J., Proc. of 9 th IEEE/CHMT Int. Electr. Manuf. Technol. Symp. (1990) 240-46.

[6] Droguet J. P., A pilot line for multichip modules substrates, Proc. of 8th European Hybrid Microelectronics Conference (1991) 280-286. 